\title{
Adapting the 3D-printed Openflexure microscope enables computational super-resolution imaging [version 1; peer
} review: 2 approved]

\author{
Stephen D. Grant (iD, Gemma S. Cairns (D), Jordan Wistuba, Brian R. Patton (iD
}

Department of Physics and SUPA, University of Strathclyde, Glasgow, UK



\section{Abstract}

We report on a 3D printed microscope, based on a design by the Openflexure project, that uses low cost components to perform fluorescence imaging. The system is sufficiently sensitive and mechanically stable to allow the use of the Super Resolution Radial Fluctuations algorithm to obtain images with resolution better than the diffraction limit. Due to the low-cost components, the entire system can be built for approximately $\$ 1200$.

\section{Keywords}

Light Microscopy , Super-resolution, Open Science , 3D Printing

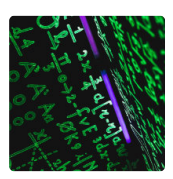

This article is included in the Mathematical,

Physical, and Computational Sciences

collection.

\author{
Open Peer Review \\ Approval Status \\ 1 \\ 2 \\ version 1 \\ 26 Nov 2019

view

view \\ 1. Richard Bowman (D), University of Bath, \\ Bath, UK \\ 2. Romain F Laine ID, University College \\ London, London, UK \\ Francis Crick Institute, London, UK \\ Any reports and responses or comments on the \\ article can be found at the end of the article.
}


Corresponding author: Brian R. Patton (brian.patton@strath.ac.uk)

Author roles: Grant SD: Formal Analysis, Supervision, Visualization, Writing - Original Draft Preparation, Writing - Review \& Editing; Cairns GS: Methodology, Resources, Validation, Writing - Review \& Editing; Wistuba J: Data Curation, Methodology, Resources; Patton BR: Conceptualization, Data Curation, Formal Analysis, Funding Acquisition, Investigation, Methodology, Project Administration, Resources, Supervision, Visualization, Writing - Original Draft Preparation, Writing - Review \& Editing

Competing interests: No competing interests were disclosed.

Grant information: This work was funded under grants from the Royal Society [CHGIR1\170017 and URFIR\180017], Engineering and Physical Sciences Research Council (EPSRC) [EP/M003701/1] and the Medical Research Council (MRC) funded SHIELD consortium [MRNO2995X/1]. G.S.C. is funded under 'OPTIMA: The EPSRC and MRC Centre for Doctoral Training in Optical Medical Imaging'. B.R.P. holds a Royal Society University Research Fellowship.

The funders had no role in study design, data collection and analysis, decision to publish, or preparation of the manuscript.

Copyright: $\odot 2019$ Grant SD et al. This is an open access article distributed under the terms of the Creative Commons Attribution License , which permits unrestricted use, distribution, and reproduction in any medium, provided the original work is properly cited.

How to cite this article: Grant SD, Cairns GS, Wistuba J and Patton BR. Adapting the 3D-printed Openflexure microscope enables computational super-resolution imaging [version 1; peer review: 2 approved] F1000Research 2019, 8:2003

https://doi.org/10.12688/f1000research.21294.1

First published: 26 Nov 2019, 8:2003 https://doi.org/10.12688/f1000research.21294.1 


\section{Introduction}

The Openflexure microscope is an open-access, 3D printable microscope which has three dimensional movement of the specimen stage provided by a flexible plastic mechanism ${ }^{1}$. It uses easily obtainable components and the fine adjustment and stability of the 3D printed microscope allows high quality optical images to be collected. Standard versions of the microscope use medium to low-resolution lenses, enabling observation of cellular features of sub-micron size.

Super-resolution radial fluctuations (SRRF) allows super resolution information to be extracted from a series of fluorescence images taken with a high numerical-aperture (NA) lens (typically NA $>0.8$ ). SRRF is a post-processing method that generates a radiality map for each frame and then looks for temporal correlations across frames to create a final image of greater resolution than the original images ${ }^{2}$. The final resolution achievable by SRRF depends on multiple factors, including the strength of the fluctuations used to generate the correlations, the labelling density and the photo-stability of the fluorophores; however, in the best-case scenario it shows resolution approaching that of localisation based super-resolution methods while utilising a wider range of hardware and fluorophores. Low illumination intensities also avoid photo-bleaching samples ${ }^{3}$.

Combining SRRF with an adapted version of the Openflexure 3D printable microscope allowed for high quality super resolution imaging on low cost hardware. SRRF analysis was carried out using Image $J^{4,5}$ plugins meaning all aspects of this work (SRRF algorithms, Microscope schematics, etc.) are freely available and open-access.

\section{Hardware design}

We give links to the designs for all key components at the end of this paper (See underlying data ${ }^{6}$ and hardware design). Measurements were performed using an adapted version of the open source 3D printable microscope (v5.17.2-LS75-M) designed by the Openflexure project. The upper image in Figure 1 shows the microscope as constructed and mounted on a $30 \times 30 \mathrm{~cm}$ aluminium breadboard for stability and portability. The colour scheme is arbitrary and does not contain any information about which parts are custom. The custom parts for this project consist of a mirror and camera holder beneath the microscope along with taller legs to allow space for the new optical path shown in the middle of Figure 1. The mirror and camera holder are combined into a single piece to control the distance from the objective to the camera and make the optical alignment more robust. This new piece is shown in the lower image of Figure 1. We also designed a cover for the camera unit to block external light and thereby improve signal to noise. The microscope was printed using polylactic acid material from Ultimaker (Red PLA - Ultimaker part no. 1618 and Green PLA - Ultimaker part no. 1608) on an Ultimaker S5 printer. As our modifications are based on a standard version of the Openflexure stage, stepper motors can be attached to the microscope if required. In this instance all stage movement and focus adjustments were made by hand with the attached adjustment gears. A compact laser module from Thorlabs (CPS532a: $4.5 \mathrm{~mW} @ 532 \mathrm{~nm}$ ) was used as the light source. This was focused using a Thorlabs plano-convex lens (LA1461-A: $\mathrm{f}=250 \mathrm{~mm}$ ), which gives a minimum spot size of $19 \mu \mathrm{m}$ at the focus. We purposely defocussed this lens to give a wider illumination spot. The excitation wavelength was chosen to allow us to image the fluorescent Nitrogen-Vacancy (NV) defect centre insamples containing nanodiamond.

A Nikon oil immersion objective (NA=1.25, 100×, Edmund Optics part number \#59-938) was used to image the sample directly this objective is not an infinity corrected lens, and so the camera is placed at the primary image plane. The objective was mounted

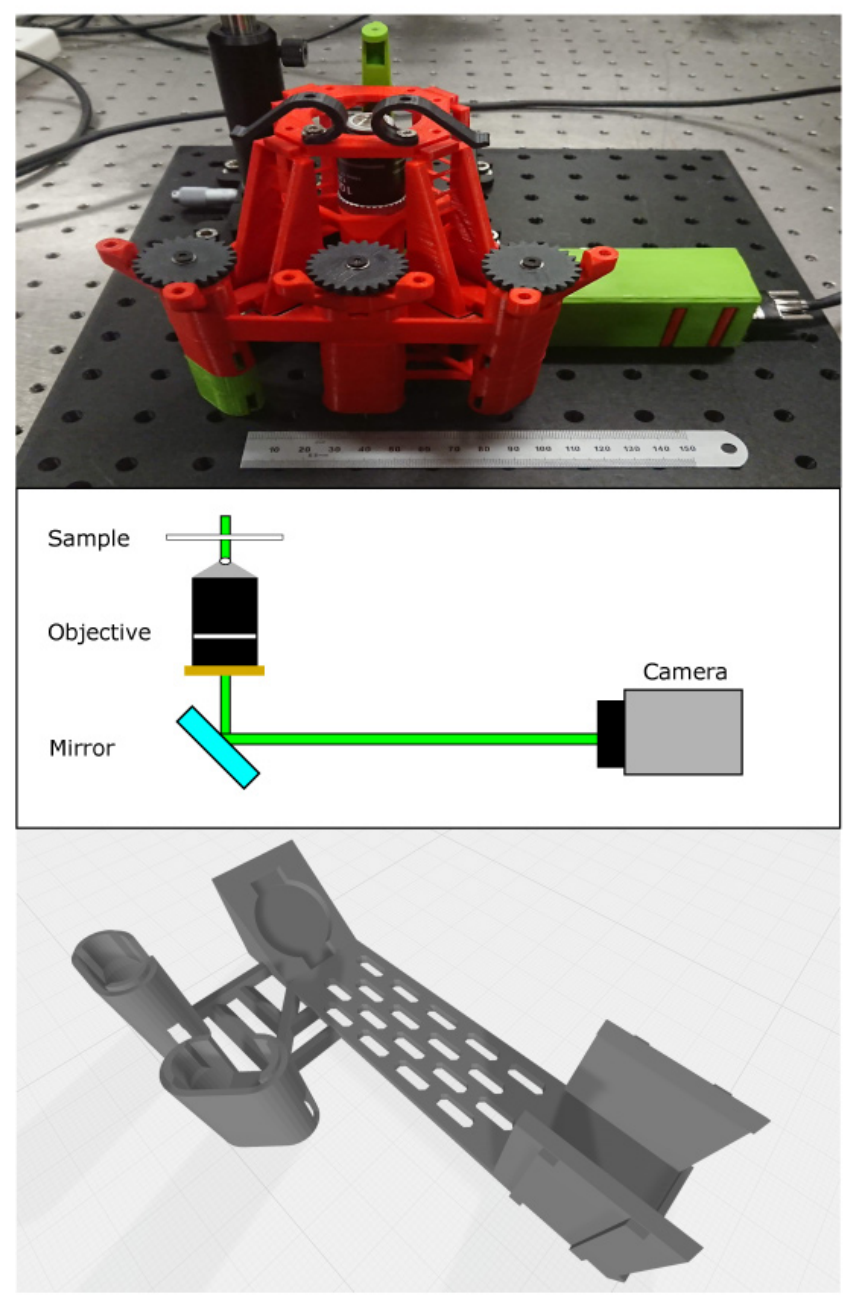

Figure 1. Layout of microscope. Top - image of microscope as constructed. It is mounted on an aluminium breadboard for portability and stability. The ruler is $15 \mathrm{~cm}$ and shown for scale. The colours of individual parts are arbitrary. Middle - beam path showing key design parameters. Illumination is from a laser mounted above the sample and the camera is mounted at the primary imaging plane of the microscope objective with an emission filter directly attached. The turning mirror allows a more compact design. Bottom: integrated mirror and camera mount to allow imaging with high sensitivity camera. We used the slots in the base of this part to pass mounting screws to the breadboard for additional stability. 
beneath the microscope stage. Focus adjustments are performed by moving the objective vertically using the adjustment gears as is standard with the Openflexure system.

A Thorlabs silver mirror (PF10-03-P01) was placed beneath the objective in our custom holder and directs the beam onto the camera. The microscope uses a UI-3060CP-M-GL camera from IDS connected to a laptop computer to capture images. The IDS camera sensor (Sony IMX174LLJ-C) has dimensions of $11.345 \mathrm{~mm} \times 7.126 \mathrm{~mm}$ with pixel size $5.86 \times 5.86 \mu \mathrm{m}$. As printed, our mirror and camera mount places the camera sensor $170 \mathrm{~mm}$ from the mounting flange of the lens. This is not in accordance with the DIN standard of $150 \mathrm{~mm}$. However, for the samples used we found that it did not create significant imaging errors but does require calibration of the image field of view at the sensor. By imaging a chrome grid sample with $10 \mu \mathrm{m}$ grid spacing, we were able to determine that we have a field of view of approximately $95.8 \times 60.2 \mu \mathrm{m}$ within the sample. This corresponds to a $49.5 \mathrm{~nm}$ pixel size at the sample; we are sampling above the Nyquist criterion for our optical system. With the known physical pixel dimensions for the camera, we therefore have a magnification of $118 \mathrm{x}$ rather than the $100 \mathrm{x}$ fromthe lens specifications.

To reject excitation laser light and allow only the emission from the NV centres to be detected, we used a 650nm longpass filter (Thorlabs FELH0650) mounted directly to the camera's c-mount hardware.

\section{Sample preparation}

We used two types of sample for this paper and their preparation is as follows:

\section{Nanodiamond on coverslips}

We used samples previously described in 7 and the following preparation protocol is from this reference. The nanodiamonds (ND) used in these experiments were produced by Adamas (Catalogue numbers ND-NV-40nm-Bio-2mg and ND-NV100nm-COOH-2ml). We prepared slides for imaging from a mix of two monodisperse suspensions (both $0.1 \% \mathrm{w} / \mathrm{v}$ ) of $40 \mathrm{~nm}$ and $100 \mathrm{~nm}$ diameter ND. The 40nm ND each contain approximately $10 \mathrm{NV}$ while there are closer to $400 \mathrm{NV}$ per $100 \mathrm{~nm}$ ND as per the manufacturers calibration information. To prepare a suspension suitable for deposition on a coverslip we first sonicated each source of NDs at room temperature for approximately 30 minutes (we have not found this step to be time critical), using a Grant Ultrasonic Bath XUBA3, to break up larger aggregates before adding $10 \mu \mathrm{l}$ of each ND suspension to $100 \mu \mathrm{l}$ of distilled water. The resulting suspension was then deposited onto a \#1.5 microscope glass cover slip and allowed to dry to ensure some ND adhered to the coverslip before being mounted on to a microscope slide using a small amount of distilled water as a mountant medium and finally sealing the sample with nail polish.

\section{Monocyte-derived macrophages}

Whole blood samples were obtained, after written informed consent, from healthy donors on the Centre for Inflammation Research Blood Resource (approved by AMREC, reference number 15-HV-013). Peripheral blood mononuclear cells (PBMCs) were first isolated from these samples before being plated onto \#1.5 square glass coverslips $(22 \times 22 \mathrm{~mm})$ in 6 well plates at a concentration of $9 \times 10^{6}$ cells/coverslip. The PBMCs were then cultured in media (RPMI (Sigma Aldrich catalogue R8758) containing 10\% low lipopolysaccharide (LPS) fetal calf serum (FBS Good Forte, Pan Biotech, P40-47500) in an incubator at $37^{\circ} \mathrm{C}$ with $5 \%$ carbon dioxide $\left(\mathrm{CO}_{2}\right)$. They were cultured for 14 days with media changed twice per week to allow for the differentiation of PBMCs into monocyte-derived macrophages (MDMs).

90nm nanodiamonds (Sigma-Aldrich 798150) were sonicated for 1 hour to break up aggregates and then added to media at a dilution of 1:100. After 14 days of cell culture, MDMs were incubated with $1 \mathrm{ml}$ of the nanodiamond/media solution at $37^{\circ} \mathrm{C}$ with $5 \% \mathrm{CO}_{2}$ for 2 days. After 2 days, media was removed from the MDMs before washing three times with pre-warmed Hank's balanced salt solution (with calcium and magnesium) to remove any remaining media. MDMs were then fixed using $2 \%$ paraformaldehyde (PFA) for 20 minutes at room temperature. Finally, the MDMs were washed three times with phosphate buffered saline (PBS) and once with deionised water $\left(\mathrm{dH}_{2} \mathrm{O}\right)$ before mounting the coverslips onto microscopy slides with ProLong Diamond Antifade Mountant(Thermofisher catalog number P36970).

\section{Data acquisition and analysis}

Data was collected using the supplied IDS software (IDS Software Suite 4.92) with gamma, auto exposure, and auto gain switched "off". Exposure time and frame rate were adjusted to produce an image with signal in the middle of the 8-bit greyscale range. Stacks of 200 images were captured as 200 frame videos. These stacks were then processed using nanoJ-Core V2.1 RC1 ${ }^{8}$ to determine the drift correction and nanoJ-SRRF v1.14Stable ${ }^{3}$ to preform super resolution radial fluctuations analysis. For the analysis of all the data presented here we used the following processing parameters within the Fiji plugins.

- Drift Correction: Averaged drift over 5 frames and corrected position to first frame

- $\quad$ SRRF: Ring radius 0.5, Radiality Magnification 6, Axes in Ring 7. Other parameters were default for plugin.

Images are presented using the Cube Helix colormap ${ }^{9}$ and were generated for publication using OriginLab Origin.

All raw (unprocessed) image data is available along with the microscope design files (Underlying data ${ }^{6}$ ).

\section{Results}

Figure 2 shows the results of imaging ND directly mounted on a coverslip. In the standard fluorescence image, Figure 2a), we see good contrast, particularly after correcting for sample drift and summing the frames. A significant increase in resolution is visible after processing the stack using the SRRF algorithm, Figure 2b). To better demonstrate the effect of the image processing, we have expanded the region in the red dashed box and present it in Figure 2c) and d). We can now clearly see individual NDs, particularly at the edges of the region highlighted. In the central region, it is likely that some of the structure shown is 

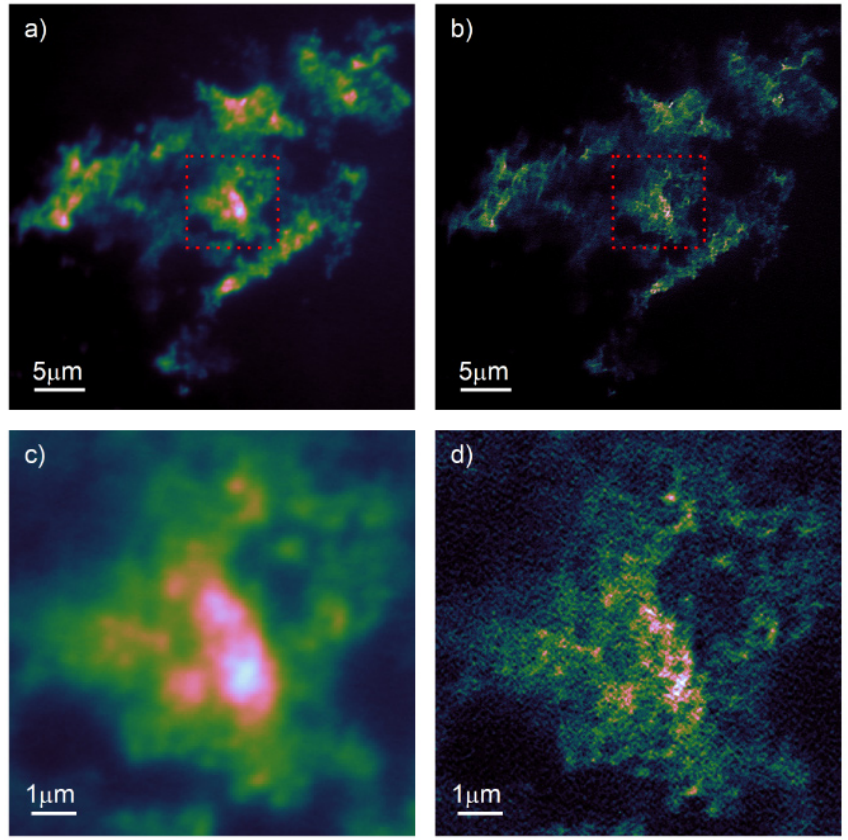

Figure 2. Fluorescence imaging of $40 \mathrm{~nm}$ and $100 \mathrm{~nm}$ nanodiamond on coverslip. a) Drift corrected and summed stack of 200 individual frames. b) Super-resolution radial fluctuations (SRRF)-processed image of same stack of images. c),d) zoom on equivalent region marked by red dashed box in $\mathbf{a}), \mathbf{b})$ showing increased resolution enabled by SRRF.

due to single ND; however, we cannot currently unambiguously rule out the presence of artefacts from the image processing algorithm.

In order to better understand the resolution enhancement, we processed the images using the Fourier Ring Correlation technique $(\mathrm{FRC})^{10}$. To estimate resolution, FRC processes pairs of images that differ only in noise: at low spatial frequencies which are dominated by the (identical) structure of the sample there is a high degree, $F R C \approx 1$, of correlation, which decreases with increasing spatial frequency until reaching a point at which the images are dominated entirely by uncorrelated noise. The spatial frequency at which this occurs is considered to be equivalent to the effective resolution of the image. There are two important caveats here:

1. The image may possess no significant features at the resolution of the system generating the image. In this case, the FRC will return an apparent resolution worse than the actual system resolution.

2. The FRC does not actually measure resolution directly, but maps spatial frequency correlations to resolution.

To consider why the second point is of relevance for our paper here, consider performing the FRC of an image, consisting entirely of random pixel values (pixel noise), with itself. In this case, the FRC would correctly return a perfect correlation at all spatial scales, and any implications on the resolution of the system would be meaningless. This incorrect resolution estimation can also arise as the signal to noise ratio (SNR) can itself influence the apparent resolution returned by the FRC algorithm. In most fluorescent microscopies, this is not a significant issue, as the fluorophores degrade and so put a limit on the maximum SNR obtainable from the sample. However, the fact that the ND emission does not degrade means that we can achieve arbitrarily high SNR by either increasing the exposure time of our sensor, or summing more sub-frames. When calculating the FRC for Figure 2a) we ran into this problem with the SNR; if you calculate the FRC by performing the correlation on the summed, drift-corrected odd versus even frames the FRC implies super-resolution imaging in wide field mode! By reducing the number of sub-frames used for the FRC, we obtained a resolution of $238 \mathrm{~nm}$, which is in line with the expected diffraction limited resolution for a wide-field microscope using a $1.25 \mathrm{NA}$ objective lens, albeit implying a higher resolution than the $280 \mathrm{~nm}$ expected for the ND emission peak at $700 \mathrm{~nm}$. An alternative approach to estimating the resolution is to measure the full width half maximum (FWHM) of individual emitters within the image. Due to the background, it is difficult to unambiguously determine the resolution in this sample. However, measuring the resolution in this manner consistently returned values for the FWHM in the range of 300-400 nm, i.e. close to diffraction limited performance.

The FRC of the SRRF processed images generated correlation curves that followed the expected behaviour for reliable resolution estimation. In this case, we recover an FRC resolution of $115 \mathrm{~nm}$. Again, this is slightly higher than the resolution returned by FWHM measurements, which tended to the range 120-140 nm. Nevertheless, it supports our assertion that the modifications to the microscope allow computational superresolution imaging using the SRRF technique.

Imaging bright, stable fluorophores that are confined to the surface of a cover slip can be considered to be the optimal sample for the characterisation of a microscope system and so somewhat unrealistic. We therefore also used ND in MDM as described above, and the resulting widefield and SRRF images are shown in Figure 3 a)-d). Again we see that the microscope performs well, generating high quality fluorescence images that process well with the SRRF algorithm. The slightly lower SNR in these images returned more reliable FRC estimates, and we see FRC resolution of $322 \mathrm{~nm}$ for the widefield images and $114 \mathrm{~nm}$ for the SRRF images.

\section{Conclusions}

SRRF imaging is possible using low cost, open-access components and software on a 3D printed microscope, achieving resolutions of $115 \mathrm{~nm}$, compared to the unprocessed (standard) resolution of $322 \mathrm{~nm}$.

When compared to traditional optical systems capable of super resolution, SRRF presents a much simpler method while the instrumentation shown here allows for its implementation at much lower costs. 

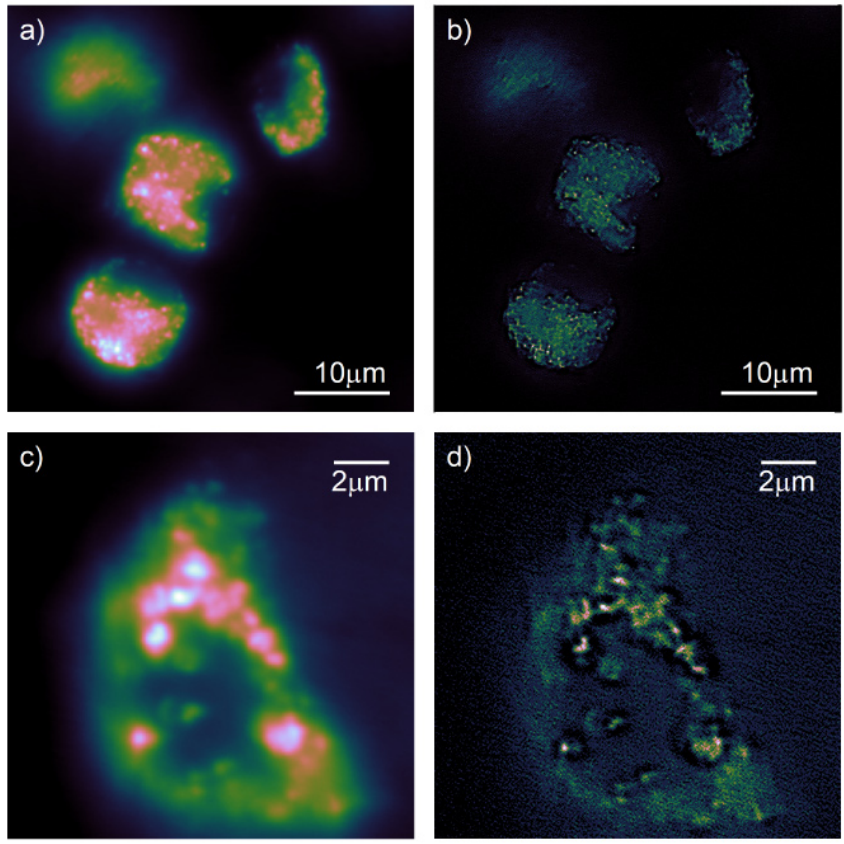

Figure 3. Fluorescence imaging of $90 \mathrm{~nm}$ nanodiamond in monocyte-derived macrophages. The nanodiamonds were suspended in the growth medium before uptake by the macrophages. a),b) large field of view encompassing multiple macrophages in both wide-field fluorescence and super-resolution radial fluctuations (SRRF) imaging modes. c),d) detail view of single macrophage in both widefield and SRRF modes.

\section{Data availability}

\section{Underlying data}

A zip file containing the 3D printable. STL files and the raw (unprocessed) data used in the images in this paper is available from the University of Strathclyde institutional repository

University of Strathclyde Knowledgebase: Data for "Adapting the 3D-printed Openflexure microscope enables computational super-resolution imaging" https://doi.org/10.15129/df032aa82b85-4ec8-adf8-ad435806b81b ${ }^{6}$

This project contains the following underlying data:

- Folder: Figure-Layout (Contains files used for Figure 1)

- BetterMicroscopeImage.JPG (A JPEG photograph of the microscope
- Cam_MirrorSupportWithLegs_V3.1.png The rendering of the custom mirror and camera unit in PNG format)

- $\quad$ Figure-LayoutV2.pdf (The file used to generate Figure 1, in PDF format)

- Figure-LayoutV2.svg (The file used to generate Figure 1, in SVG format)

- $\quad$ Folder: Figure NV (Contains files used for Figure 2)

- FigureNDonCoverslip4.pdf (The file used to generate Figure 2 in PDF format)

- $\quad$ FigureNDonCoverslip4.png (The file used to generate Figure 2 in PNG format)

- NV200Frames4.avi (The movie (in AVI format) containing the raw data for Figure 2)

- Folder: Figure-Macrophages (Contains files used for Figure 3)

- Figure-Macrophages.png (The file used to generate Figure 3, in PNG format)

- MacrophageFrames3.avi (The movie (in AVI format) containing the raw data for Figure 3)

- Folder: Microscope STL Files (This folder contains multiple stl files that can be directly printed on a 3D printer that can print files in this widely used file format. All parts should be printed and assembled using the instructions on the https://openflexure.org/ website, along with the additional details given in this paper)

All figures and raw data are available under the terms of the Creative Commons Attribution 4.0 International license (CC-BY 4.0).

The .stl files for the 3D printer, including the new components described in this paper, are available under the terms of the CERN Open Hardware licence (CERN OHL).

\section{Hardware design}

The microscope was constructed using an adapted version of the open source 3D printable microscope (v5.17.2-LS75-M) designed by the Openflexure project, https://openflexure.org/, with modifications as described above.
1. Sharkey JP, Foo DC, Kabla A, et al.: A one-piece 3D printed flexure translation stage for open-source microscopy. Rev Sci Instrum. 2016; 87(2): 025104. PubMed Abstract | Publisher Full Text

2. Gustafsson N, Culley S, Ashdown G, et al.: Fast live-cell conventional fluorophore nanoscopy with ImageJ through super-resolution radial fluctuations. Nat Commun. 2016; 7(1): 12471.

PubMed Abstract | Publisher Full Text | Free Full Text
3. Culley S, Tosheva KL, Matos Pereira P, et al.: SRRF: Universal live-cell superresolution microscopy. Int J Biochem Cell Biol. 2018; 101: 74-79. PubMed Abstract | Publisher Full Text | Free Full Text

4. Rueden $\mathrm{CT}$, Schindelin J, Hiner MC, et al.: ImageJ2: ImageJ for the next generation of scientific image data. BMC Bioinformatics. 2017; 18(1): 529. PubMed Abstract | Publisher Full Text | Free Full Text

5. Schindelin J, Arganda-Carreras I, Frise E, et al.: Fiji: an open-source platform for 
biological-image analysis. Nat Methods. 2012; 9(7): 676-682. PubMed Abstract | Publisher Full Text | Free Full Text

6. Patton B: Data for: "Adapting the 3D-printed Openflexure microscope enables computational super-resolution imaging". 2019.

http://www.doi.org/10.15129/df032aa8-2b85-4ec8-adf8-ad435806b81b

7. Johnstone GE, Cairns GS, Patton BR: Nanodiamonds enable adaptive-optics enhanced, super-resolution, two-photon excitation microscopy. $R$ Soc Open Sci. 2019; 6(7): 190589.

PubMed Abstract | Publisher Full Text | Free Full Text
Laine RF, Tosheva KL, Gustafsson N, et al.: NanoJ: A high-performance open-source super-resolution microscopy toolbox. J Phys D Appl Phys. 2019; 52(16): 163001 Publisher Full Text

9. Green DA: A colour scheme for the display of astronomical intensity images. Bull Astr Soc India. 2011; 39: 289-295.

Reference Source

10. Nieuwenhuizen RP, Lidke KA, Bates M, et al.: Measuring image resolution in optical nanoscopy. Nat Methods. 2013; 10(6): 557-562.

PubMed Abstract | Publisher Full Text | Free Full Text 


\section{Open Peer Review}

\section{Current Peer Review Status:}

\section{Version 1}

Reviewer Report 26 May 2020

https://doi.org/10.5256/f1000research.23450.r63807

(C) $\mathbf{2 0 2 0}$ Laine $\mathbf{R}$. This is an open access peer review report distributed under the terms of the Creative Commons Attribution License, which permits unrestricted use, distribution, and reproduction in any medium, provided the original work is properly cited.

\section{Romain F Laine}

1 University College London, London, UK

2 Francis Crick Institute, London, UK

Grant et al. provides a clever open-source microscopy platform capable of SRRF super-resolution microscopy. Open-source and cheap microscopy developments are currently a hot topic in the field and a number of contributions are available from various labs across the world. My expertise is in the development of Super-resolution microscopy techniques, including hardware and software-based solutions. This includes the SRRF super-resolution technique and I currently work in the lab (Henriques lab) which pioneered the method.

Typical open-source microscopes designed for high resolution can suffer from a number of drawbacks:

1. Mechanical stability can be poor and compromise the image acquisition by adding motion blur and sample drift

2. Use of typical high NA microscope objectives can be expensive and require alignment of full optical train (objective, tube lens, camera)

3. Sample movement and focus control can be either bulky and/or expensive The implementation of Grant et al. nicely combines a number of open-source components (3D printed elements, simple opto-mechanics and cheap CMOS camera) that makes it uniquely simple and powerful and provide interesting trade-offs for these common drawbacks:

Use of the cheap and flexible OpenFlexure system for sample movement control and objective focus control

Simple, single 3D-printed block assembly for the detection path of the fluorescence microscope

Use of finite conjugate objective, doing away with tube lens altogether and simplifying significantly the assembly

The super-resolution capability is enabled by SRRF, an approach based on picking up on radial 
symmetry of single emitter fluorescence pattern and correlated temporal fluctuations of these emitters. I do not know about the photophysics of NV and their blinking properties but a preprint from Narayanasamy et al. ${ }^{1}$ shows their compatibility with super-resolution microscopy. Furthermore, the resolution improvement quoted and the images shown here are convincing.

Using SRRF as a super-resolution microscopy technique is a great way to address the aspect of drift since SRRF requires only relatively short acquisition times compared to other super-resolution microscopy techniques such as SMLM and also allows the correction of sample drift at postacquisition level. Although SRRF does not provide as high a resolution as single-molecule localization microscopy (SMLM), the short acquisition times are more compatible with the plastic stage of OpenFlexure, which may introduce more sample drift than typical metal body assemblies.

I agree however with Reviewer 1 that some measurements of stability and amplitude of drift on the order of a typical acquisition would be helpful. This could be easily done on single emitters such as commonly used TetraSpeck beads or the single emitter NV that the authors describe in the paper.

The use of a finite objective (which is surprisingly cheap: $£ 203$ as of May 2020) with a high NA and a decent plan field of view for the price provides a great compromise between price and performance. This work constitutes the first use of finite conjugate objective to super-resolution microscopy to my knowledge.

I am especially impressed by the quote of a FWHM of 300-400 nm on single emitters, especially since the objective is not quite working at the nominal conjugate. Is there any reason why the system was not designed to be at the nominal conjugate distance? It would be interesting to get some insight into some of the design choices made here for whoever would like to replicate the system (which I may very well do myself at some point in the future).

The discussion about FRC is absolutely valid and is a nice addition to the overall idea of the paper. A few additional comments however:

It is true (as reviewer \#1 highlighted) that the illumination system is described only superficially, yet it is unavoidable to build an efficient illumination system for a good quality fluorescence microscopy. Also, the downside of using an illumination configuration coming from the top of the objective and therefore contributing to a larger amount of background light onto the detector should be discussed/mentioned

Since one of the novelty is the use of finite conjugate objective, it would be interesting to characterise it a little further in terms of PSF size and shape (as preliminary done), flatness of the field, sensitivity (I suspect that transmission is poorer than high end objectives) and multi-colour capability (although the latter might be out of scope)

Is the rationale for developing the new method (or application) clearly explained? Yes, it is a nice set of trade-offs introduced here, truly reducing the cost of high resolution microscope to the bare minimum, taking advantage of 3D printing especially, which I have not seen in other open-source microscopy implementations out there.

Is the description of the method technically sound?

Yes, the authors present a couple of experiments showing proof-of-concept and some performance. 
Are sufficient details provided to allow replication of the method development and its use by others?

Yes, generally. STL files and other technical details are all there. Maybe a bit more info about how the illumination was set up would be nice for completeness.

If any results are presented, are all the source data underlying the results available to ensure full reproducibility?

Yes.

Are the conclusions about the method and its performance adequately supported by the findings presented in the article?

Yes, apart from the couple of suggestions of characterisation of mechanical stability and microscope objective performance which would really nail down a full characterisation of the system presented here.

\section{References}

1. Narayanasamy K, Price J, Mesquita-Riberio R, Mather M, et al.: Self-activated photoblinking of nitrogen vacancy centers in nanodiamonds (sandSTORM): A method for rapid single molecule localization microscopy with unlimited observation time. bioRxiv. 2020. Publisher Full Text

Is the rationale for developing the new method (or application) clearly explained?

Yes

Is the description of the method technically sound?

Yes

Are sufficient details provided to allow replication of the method development and its use by others?

Yes

If any results are presented, are all the source data underlying the results available to ensure full reproducibility?

Yes

Are the conclusions about the method and its performance adequately supported by the findings presented in the article?

Yes

Competing Interests: No competing interests were disclosed.

Reviewer Expertise: My expertise is in the development of Super-resolution microscopy techniques, including hardware and software-based solutions. This includes the SRRF superresolution technique and I currently work in the lab (Henriques lab) which pioneered the method.

I confirm that I have read this submission and believe that I have an appropriate level of 


\section{expertise to confirm that it is of an acceptable scientific standard.}

Reviewer Report 14 January 2020

https://doi.org/10.5256/f1000research.23450.r58499

(c) 2020 Bowman R. This is an open access peer review report distributed under the terms of the Creative Commons Attribution License, which permits unrestricted use, distribution, and reproduction in any medium, provided the original work is properly cited.

\section{Richard Bowman}

Centre for Photonics and Photonic Materials, Department of Physics, University of Bath, Bath, UK

This article describes an open, low-cost, super-resolution imaging system, using nanodiamond probes and the SRRF algorithm to localise individual emitters. It uses the OpenFlexure Microscope as the critical optomechanical mount, responsible for holding the microscope objective and the sample - consequently, most of the mounting and micropositioning hardware in the system can be 3D printed from open, documented designs; the major expenses are the camera, microscope objective, and laser - totalling around $\$ 1200$.

I have led the OpenFlexure Microscope project for some years now, and am very excited to see it used for super-resolution imaging. It is particularly pleasing that this imaging is also enabled by open-source software in the form of ImageJ, nanoJ-Core, and nanoJ-SRRF. The authors have done a great job of producing a clear, concise manuscript that describes the system and their characterisation data, that proves the principle of accessible, DIY super-resolution microscopy. I am very happy to endorse the manuscript as scientifically sound.

I should be clear about what I don't know; I can't claim to be an expert on super-resolution techniques, and so I'm not able to state authoritatively whether I would trust the FRC figures. The authors are commendably clear about the fact that FRC can be confused by excessively high signal-to-noise ratios. While they do state the FRC figures, they also provide the more direct FWHM measure of the point spread function. FWHM gives only slightly worse resolution figures than FRC; the system is still clearly beating the diffraction limit by some margin.

Overall, I am delighted to see this manuscript, which really advances what is possible using an inexpensive, home-built microscope. I very much hope that others are able to build on this development, and use some of the techniques shown to make novel microscopy instruments accessible, as well as reproducible.

I have detailed below a few minor improvements to the manuscript that might help someone reproducing the system. I don't believe any of these affect scientific soundness, but they would nonetheless be helpful.

First paragraph of "hardware design": "insamples" is missing a space in the PDF version. There's also a "fromthe" later on, though both are OK in the web view.

Figure 1: it would be nice to add the laser diode and emission filter to the middle panel. I 
understand the laser enters the sample from the top, and it is stated in the text that the emission filter is mounted to the camera's C-mount hardware - but it would be clearer if they appeared in the diagram. Also, I don't think it's stated anywhere how the laser diode is mounted - is that the half-inch post I see in the background of the photo? While it's hardly a scientifically critical detail, it's helpful for anyone reproducing the system.

Sample Preparation, final paragraph: were the $90 \mathrm{~nm}$ nanodiamonds functionalised in any way to label specific features of the MDMs? I don't think that's specified if so. If not, do you know where they tend to localise within the cell? It doesn't affect the imaging performance either way, but I'm curious.

Data acquisition and analysis: it would be nice to know the total elapsed time of the 200-frame video, and ideally the exposure time if that's available. A comment on how far the stage drifted laterally over that time might also be helpful (particularly because there will, presumably, be axial drift that is not corrected for). Do the authors believe drift was the limiting factor in the resolution that was achieved?

Reference [1] is the most up-to-date paper on the OpenFlexure Microscope currently published, and the authors are absolutely correct in referencing openflexure.org as the most up to date information on the project. However, we have described the microscope recently in a pre-print and I have taken the liberty of including the DOI below ${ }^{1}$. This version of the microscope is much closer to the one used in the present article than the earlier version described by Sharkey et al. ${ }^{2}$.

\section{References}

1. Collins J, Knapper J, Stirling J, Mduda J, et al.: Robotic microscopy for everyone: the OpenFlexure Microscope. bioRxiv. 2019. Publisher Full Text

2. Sharkey JP, Foo DC, Kabla A, Baumberg JJ, et al.: A one-piece 3D printed flexure translation stage for open-source microscopy.Rev Sci Instrum. 2016; 87 (2): 025104 PubMed Abstract | Publisher Full Text

Is the rationale for developing the new method (or application) clearly explained? Yes

Is the description of the method technically sound?

Yes

Are sufficient details provided to allow replication of the method development and its use by others?

Yes

If any results are presented, are all the source data underlying the results available to ensure full reproducibility?

Yes

Are the conclusions about the method and its performance adequately supported by the findings presented in the article? 
Yes

Competing Interests: I lead the OpenFlexure Project, including a financial interest in OpenFlexure Industries Ltd. OpenFlexure Industries exists with the intention of making the OpenFlexure Microscope easier to obtain, but does not hold any exclusive licenses. While I am delighted to see the project used in this way, I have not been involved in any of the work described, and do not stand to make a financial profit directly or indirectly as a result.

Reviewer Expertise: My expertise is in optical instrumentation, particularly microscope design and optomechanics. I am not an expert in superresolution, as stated in my report, but am comfortable assessing the instrumentation aspects of the work.

I confirm that I have read this submission and believe that I have an appropriate level of expertise to confirm that it is of an acceptable scientific standard.

The benefits of publishing with F1000Research:

- Your article is published within days, with no editorial bias

- You can publish traditional articles, null/negative results, case reports, data notes and more

- The peer review process is transparent and collaborative

- Your article is indexed in PubMed after passing peer review

- Dedicated customer support at every stage

For pre-submission enquiries, contact research@f1000.com 